Pacific Journal of Mathematics

ON THE COMPUTATIONS OF THE NIELSEN NUMBER 


\title{
ON THE COMPUTATION OF THE NIELSEN NUMBER
}

\author{
BOJU JIANG
}

The aim of this note is to show the following

MAIN THEOREM. Let $X$ be a compact connected $A N R, f: X \rightarrow X$ be a map. Suppose there is an integer $n$ such that $f_{\pi}^{n}\left(\pi_{1}(X)\right) \subset J\left(f^{n}\right)$. Then any two fixed point classes of $f$ have the same index. Hence

$L(f)=0$ implies $N(f)=0$, while

$L(f) \neq 0$ implies $N(f)=\#$ Coker $\left(H_{1}(X) \stackrel{1-f}{\rightarrow}{ }^{*} H_{1}(X)\right)$.

Here $L(f)$ and $N(f)$ are the Lefschetz number and Nielsen number of $f$ respectively, and $J(F) \subset \pi_{1}(X)$ stands for the Jiang subgroup of $f$.

This is an improvement of a previous theorem ([3], see also [1]) which required the stronger hypothesis $J(f)=\pi_{1}(X)$. A nice feature of this improvement is that the present hypothesis is symmetric, in the sense that given $\varphi: X \rightarrow Y$ and $\psi: Y \rightarrow X$, then $\psi \circ \varphi: X \rightarrow X$ satisfies the condition iff $\varphi \circ \psi: Y \rightarrow Y$ does (see $\S 5$ ). The symmetry between $\psi \circ \varphi$ and $\varphi \circ \psi$ is a built-in symmetry of the fixed point problem. It occurs at both the geometric level, because the fixed point sets of $\psi \circ \varphi$ and $\varphi \circ \psi$ are homeomorphic, and at the algebraic level: $N(\psi \circ \varphi)=N(\varphi \circ \psi)$. So, a theorem which respects this symmetry is better than one which does not.

The proof of the main theorem given in $\S 3$ is based on an analysis of the relationship between the fixed point classes of $f$ and those of the iterates of $f(\S 2)$. Our notation and terminology is explained in $\S 1$. Some corollaries are given in $\S 4$.

The mod $p$ Index Theorem in $\$ 2$ was known to Ross Geoghegan, and is implicit in [2]. The author is grateful to him for a discussion about it. The proof given here is based on an Approximation Theorem which is of independent interest.

1. Notation and terminology. We will use the notation of [4]. For the convenience of the reader, we give a brief review of the basic notions in the theory of fixed point classes. Cf. [1], [5], [4].

Let $X$ be a compact connected ANR, and let $p: \tilde{X} \rightarrow X$ be its universal covering space. Let $\pi$ be the group of covering transformations, identified with $\pi_{1}\left(X, x_{0}\right)$ as usual, where $x_{0}$ is a prescribed base point of $X$. Let $f: X \rightarrow X$ be a map. Letting $\tilde{f}: \tilde{X} \rightarrow \tilde{X}$ be a lifting of $f$, then every 
lifting of $f$ can be uniquely written as $\alpha \circ \tilde{f}, \alpha \in \pi$. The lifting $\tilde{f}$ determines an endomorphism $\tilde{f}_{\pi}: \pi \rightarrow \pi$ by $\tilde{f}_{\pi}(\alpha) \circ \tilde{f}=\tilde{f} \circ \alpha, \alpha \in \pi$. The relation between $\tilde{f}_{\pi}$ and the induced homomorphism $f_{\pi}: \pi_{1}\left(X, x_{0}\right) \rightarrow$ $\pi_{1}\left(X, f\left(x_{0}\right)\right)$ is that, for any path $\tilde{w}$ in $\tilde{X}$ from a point $\tilde{x}_{0} \in p^{-1}\left(x_{0}\right)$ to $\tilde{f}\left(\tilde{x}_{0}\right)$, we have $\tilde{f}_{\pi}=w_{*} \circ f_{\pi}$, where $w_{*}: \pi_{1}\left(X, f\left(x_{0}\right)\right) \rightarrow \pi_{1}\left(X, x_{0}\right)$ is the isomorphism induced by the path $w=p \circ \tilde{w}$.

Two liftings $\tilde{f}, \tilde{f}^{\prime}$ of $f$ are conjugate iff there is a $\gamma \in \pi$ such that $\tilde{f}^{\prime}=\gamma \circ \tilde{f} \circ \gamma^{-1}$. Hence $\alpha \circ \tilde{f}$ and $\beta \circ \tilde{f}$ are conjugate iff $\alpha$ and $\beta$ are $\tilde{f}_{\pi}$-conjugate, i.e. there is a $\gamma \in \pi$ such that $\beta=\gamma \alpha \tilde{f}_{\pi}\left(\gamma^{-1}\right)$. Each conjugacy class of liftings $[\alpha \circ \tilde{f}]$ determines a fixed point class $p \operatorname{Fix}(\alpha \circ \tilde{f})$ of $f$. The index of a fixed point class $\mathbf{F}=p \operatorname{Fix}(\alpha \circ \tilde{f})$ is denoted by index $(f, \mathbf{F})$.

The homotopy invariance of the index: If a homotopy $\left\{h_{t}\right\}: X \rightarrow X$ lifts to a homotopy $\left\{\tilde{h_{t}}\right\}: \tilde{X} \rightarrow \tilde{X}$, then we say $\mathbf{F}_{0}=p \operatorname{Fix}\left(\tilde{h}_{0}\right)$ corresponds to $\mathbf{F}_{1}=p \operatorname{Fix}\left(\tilde{h}_{1}\right)$ under $\left\{h_{t}\right\}$, and we have

$$
\operatorname{index}\left(h_{0}, \mathbf{F}_{0}\right)=\operatorname{index}\left(h_{1}, \mathbf{F}_{1}\right) \text {. }
$$

The commutativity of the index: If $\varphi: X \rightarrow Y$ and $\psi: Y \rightarrow X$, then for any fixed point class $\mathbf{F}$ of $f=\psi \circ \varphi, \mathbf{G}=\varphi \mathbf{F}$ is also a fixed point class of $g=\varphi \circ \psi$, and we have

$$
\operatorname{index}(f, \mathbf{F})=\operatorname{index}(g, \mathbf{G}) .
$$

More generally, if $U$ is open in $X$ and has no fixed points on its boundary, we have

$$
\operatorname{index}(f, U)=\operatorname{index}\left(g, \psi^{-1}(U)\right) \text {. }
$$

The subgroup $J\left(f, x_{0}\right) \subset \pi_{1}\left(X, f\left(x_{0}\right)\right)$ is defined to consist of loop classes $\alpha^{\prime}$ such that there is a cyclic homotopy $\left\{h_{t}\right\}: f \simeq f$ of which the trace $\left\{h_{t}\left(x_{0}\right)\right\}$ is in $\alpha^{\prime}$. Similary the subgroup $J(\tilde{f}) \subset \pi$ is defined as the subgroup of elements $\alpha$ such that there is a cyclic homotopy $\left\{h_{t}\right\}: f \simeq f$ which lifts to $\left\{\tilde{h}_{t}\right\}: \tilde{f} \simeq \alpha \circ \tilde{f}$. The subgroups are related by the isomorphism $w_{*}$ above: $J(\tilde{f})=w_{*} J\left(f, x_{0}\right)$. Hence the condition $f_{\pi} \pi_{1}\left(X, x_{0}\right) \subset$ $J\left(f, x_{0}\right)$ is equivalent to $\tilde{f}_{\pi} \pi \subset J(\tilde{f})$. This condition is independent of the choice of the base point $x_{0}$, so we may write $f_{\pi} \pi_{1}(X) \subset J(f)$. The elements of $\tilde{f}_{\pi} \pi$ commute with elements of $J(\tilde{f})$ (cf. [3] Lemma 4.4), so if $\tilde{f}_{\pi} \pi \subset J(\tilde{f})$ then $\tilde{f}_{\pi} \pi$ is abelian. If $\varphi: X, x_{0} \rightarrow Y, y_{0}$ and $\psi: Y, y_{0} \rightarrow X, x_{0}$, then it is not hard to see that $\varphi_{\pi} J\left((\psi \circ \varphi)^{n}, x_{0}\right) \subset J\left((\varphi \circ \psi)^{n+1}, y_{0}\right)$.

In $\$ 2$ we will need the following technical notion. An affine map $g$ : $\sigma \rightarrow \tau$ sending a $k$-simplex $\sigma$ onto a $k$-simplex $\tau$ is said to be expanding if $d\left(g(x), g\left(x^{\prime}\right)\right)>d\left(x, x^{\prime}\right)$ whenever $x \neq x^{\prime}$. If the diameter of $\sigma$ is less than the distance between the barycenter and the boundary of $\tau$, then $g$ is certainly expanding. If $\sigma \subset \tau$, the affine map $g: \sigma \rightarrow \tau$ is expanding, and $g$ 
has a fixed point $x_{0}$ in the interior of $\sigma$, then $x_{0}$ is the only fixed point on $\sigma$ and the index of $g$ at $x_{0}$ equals $(-1)^{k}$ times the sign of the determinant of $g$; in symbols,

$$
\operatorname{index}\left(g, x_{0}\right)=(-1)^{k} \operatorname{sgn} \operatorname{det} g \text {. }
$$

2. Fixed point classes of iterates of $f$. Let $n$ be a natural number. We write $\tilde{f}^{(n)}$ for an arbitrary lifting of the iterate $f^{n}: X \rightarrow X$, and $\tilde{f}^{n}$ for the iterate of a lifting $\tilde{f}$ of $f$. It is obvious that $p \operatorname{Fix}(\tilde{f}) \subset p \operatorname{Fix}\left(\tilde{f}^{n}\right)$. We define $p \operatorname{Fix}\left(\tilde{f}^{n}\right)$ to be the fixed point class of $f^{n}$ containing $p \operatorname{Fix}(\tilde{f})$. Thus, each fixed point class (empty or not) of $f$ is contained in a unique fixed point class of $f^{n}$. (Note that if $p \operatorname{Fix}(\tilde{f})$ is empty, it is contained in every fixed point class of $f^{n}$ in the set theoretical sense.) For the fixed point class $p \operatorname{Fix}(\alpha \circ \tilde{f})$, the containing fixed point class of $f^{n}$ is $p \operatorname{Fix}(\alpha \circ \tilde{f})^{n}=$ $p \operatorname{Fix}\left(\alpha^{(\mathrm{n})} \circ \tilde{f}^{n}\right)$, where $\alpha^{(n)}=\alpha \tilde{f}_{\pi}(\alpha) \cdots \tilde{f}_{\pi}^{n-1}(\alpha)$, since

$$
\begin{aligned}
\alpha \circ \tilde{f} \circ \cdots \circ \alpha \circ \tilde{f} \circ \alpha \circ \tilde{f} & =\alpha \circ \tilde{f} \circ \cdots \circ\left(\alpha \tilde{f}_{\pi}(\alpha)\right) \circ \tilde{f} \circ \tilde{f}=\cdots \\
& =\left(\alpha \tilde{f}_{\pi}(\alpha) \cdots \tilde{f}_{\pi}^{n-1}(\alpha)\right) \circ \tilde{f} \circ \cdots \circ \tilde{f} \circ \tilde{f} .
\end{aligned}
$$

Every $\tilde{f}^{(n)}$ can be factored as $\tilde{f}_{n} \circ \ldots \circ \tilde{f}_{2} \circ \tilde{f}_{1}$, where $\tilde{f}_{l}$ are liftings of $f, i=1, \ldots, n$. It is obvious that

$$
\tilde{f}_{1} \operatorname{Fix}\left(\tilde{f}_{n} \circ \cdots \circ \tilde{f}_{2} \circ \tilde{f}_{1}\right)=\operatorname{Fix}\left(\tilde{f}_{1} \circ \tilde{f}_{n} \circ \cdots \circ \tilde{f}_{2}\right),
$$

and

$$
f\left(p \operatorname{Fix}\left(\tilde{f}_{n} \circ \cdots \circ \tilde{f}_{2} \circ \tilde{f}_{1}\right)\right)=p \operatorname{Fix}\left(\tilde{f}_{1} \circ \tilde{f}_{n} \circ \cdots \circ \tilde{f}_{2}\right) .
$$

We say that the fixed point class $p \operatorname{Fix}\left(\tilde{f}_{1} \circ \tilde{f}_{n} \circ \cdots \circ \tilde{f}_{2}\right)$ is the f-image of the fixed point class $p \operatorname{Fix}\left(\tilde{f}_{n} \circ \cdots \circ \tilde{f}_{2} \circ \tilde{f}_{1}\right)$. It is easy to see that if $\tilde{f}_{n}^{\prime} \circ \cdots \circ \tilde{f}_{2}^{\prime} \circ \tilde{f}_{1}^{\prime}$ is conjugate to $\tilde{f}_{n} \circ \cdots \circ \tilde{f}_{2} \circ \tilde{f}_{1}$, then $\tilde{f}_{1}^{\prime} \circ \tilde{f}_{n}^{\prime} \circ \cdots \circ \tilde{f}_{2}^{\prime}$ is conjugate to $\tilde{f}_{1} \circ \tilde{f}_{n} \circ \cdots \circ \tilde{f}_{2}$, so the definition of $f$-image is independent of the factorization of $\tilde{f}^{(n)}$. It follows from the commutativity of the index that for any fixed point class $\mathbf{F}^{(n)}=p \operatorname{Fix}\left(\tilde{f}_{n} \circ \cdots \circ \tilde{f}_{2} \circ \tilde{f}_{1}\right)$ of $f^{n}$ we have

$$
\text { index }\left(f^{n}, \mathbf{F}^{(n)}\right)=\operatorname{index}\left(f^{n}, f \mathbf{F}^{(n)}\right)
$$

because $f \circ f^{n-1}=f^{n-1} \circ f$. Note that if $\mathbf{F}^{(n)}$ contains a fixed point class of $f$, i.e. $\mathbf{F}^{(n)}=p \operatorname{Fix}\left(\tilde{f}^{n}\right)$ for some lifting $\tilde{f}$ of $f$, then by the above definition of the $f$-image we have $f \mathbf{F}^{(n)}=\mathbf{F}^{(n)}$.

Lemma. (i) Suppose $\left\{h_{t}\right\}: X \rightarrow X$ is a homotopy. Let $\mathbf{F}_{0}, \mathbf{F}_{1}, \mathbf{F}_{0}^{(n)}, \mathbf{F}_{1}^{(n)}$ be fixed point classes of $h_{0}, h_{1}, h_{0}^{n}, h_{1}^{n}$ respectively. If $\mathbf{F}_{0} \subset \mathbf{F}_{0}^{(n)}, \mathbf{F}_{1} \subset \mathbf{F}_{1}^{(n)}$, and $\mathbf{F}_{0}$ corresponds to $\mathbf{F}_{1}$ under $\left\{h_{t}\right\}$, then $\mathbf{F}_{0}^{(n)}$ corresponds to $\mathbf{F}_{1}^{(n)}$ under $\left\{h_{t}^{n}\right\}$. 
(ii) Suppose $\varphi: X \rightarrow Y, \psi: Y \rightarrow X$. Let $\mathbf{F}, \mathbf{G}, \mathbf{F}^{(n)}, \mathbf{G}^{(n)}$ be fixed point classes of $\psi \circ \varphi, \varphi \circ \psi,(\psi \circ \varphi)^{n},(\varphi \circ \psi)^{n}$ respectively. If $\mathbf{F} \subset \mathbf{F}^{(n)}, \mathbf{G} \subset \mathbf{G}^{(n)}$ and $\mathbf{G}=\varphi \mathbf{F}$, then $\mathbf{G}^{(n)}=\varphi \mathbf{F}^{(n)}$.

The proof is left to the reader.

The mod $p$ INDEX TheOREM. Suppose $X$ is a compact connected ANR, $f: X \rightarrow X$ is a map. Suppose $n=p^{r}, p=$ prime. Let $\mathbf{F}^{(n)}$ be a fixed point class of $f^{n}$ such that $\mathbf{F}^{(n)}=f \mathbf{F}^{(n)}$. Then

$$
\operatorname{index}\left(f^{n}, \mathbf{F}^{(n)}\right) \equiv \sum_{\mathbf{F}} \operatorname{index}(f, \mathbf{F}) \bmod p,
$$

where the summation is over all fixed point classes $\mathbf{F}$ of $f$ contained in $\mathbf{F}^{(n)}$.

Proof. Suppose $X$ is dominated by a fintie simplicial polyhedron $Y=|K|$, with $\varphi: X \rightarrow Y$ and $\psi: Y \rightarrow X$ such that $\psi \circ \varphi \simeq \mathrm{id}_{X}$. Then $f \simeq(f \circ \psi) \circ \varphi: X \rightarrow X$. By the homotopy invariance and the commutativity of the index, and the above Lemma, it is readily seen that the theorem for $f$ follows from the theorem for $g=\varphi \circ(f \circ \psi): Y \rightarrow Y$. So without loss we may assume $X$ to be a polyhedron $|K|$.

In view of the Approximation Theorem below, we may assume further that $f$ is a simplicial map $K^{\prime} \rightarrow K$ such that

(i) every fixed point of $f$ lies in the interior of some maximal simplex of $K^{\prime}$ on which $f$ is expanding, and

(ii) $f^{n}$ has only a finite number of fixed points.

Let $\mathbf{F}_{i}$ be the fixed point classes of $f$ contained in $\mathbf{F}^{(n)}$. Since $f \mathbf{F}^{(n)}=$ $\mathbf{F}^{(n)}$, then $\mathbf{F}^{(n)}-\cup_{i} \mathbf{F}_{i}$ is invariant under $f$ and decomposes into orbits. Each orbit length divides $n=p^{r}$, hence is divisible by the prime $p$. But by commutativity

$$
\operatorname{index}\left(f^{n}, x\right)=\operatorname{index}\left(f^{n}, f(x)\right)
$$

for every isolated fixed point $x$ of $f^{n}$, hence we have

$$
\operatorname{index}\left(f^{n}, \mathbf{F}^{(n)}\right) \equiv \sum_{i} \operatorname{index}\left(f^{n}, \mathbf{F}_{i}\right) \bmod p .
$$

For each $x \in \mathbf{F}_{i}, f$ (hence $f^{n}$ ) is an expanding affine map in a $k$-dimensional Euclidean neighborhood of $x$. Thus

$$
\begin{aligned}
\text { index }(f, x) & =(-1)^{k} \text { sgn det } f, \\
\text { index }\left(f^{n}, x\right) & =(-1)^{k} \text { sgn det } f^{n} .
\end{aligned}
$$


So

$$
\begin{aligned}
& \text { index }\left(f^{n}, x\right)=\operatorname{index}(f, x) \quad \text { if } p \neq 2 \text {, } \\
& \text { index }\left(f^{n}, x\right) \equiv \operatorname{index}(f, x) \quad \bmod 2 \text { if } p=2 \text {. }
\end{aligned}
$$

In any case

$$
\operatorname{index}\left(f^{n}, x\right) \equiv \operatorname{index}(f, x) \bmod p
$$

Hence

$$
\operatorname{index}\left(f^{n}, \mathbf{F}_{i}\right) \equiv \operatorname{index}\left(f, \mathbf{F}_{i}\right) \bmod p
$$

The Approximation Theorem mentioned in the above proof follows.

ApProximation TheOrem. Let $K$ be a finite simplicial complex, $f$ : $|K| \rightarrow|K|$ be a map. Then there is a subdivision $K^{\prime}$ of $K$ and a simplicial map $g: K^{\prime} \rightarrow K$ homotopic to $f$ such that

(i) every fixed point of $g$ lies in the interior of some maximal simplex of $K^{\prime}$ on which $g$ is expanding, and

(ii) every iterate $g^{n}$ of $g$ has only a finite number of fixed points.

Proof. Each simplex of $K$ of positive dimension has a nonzero distance between its barycenter and its boundary. Let $\varepsilon$ be the shortest of such distances. Let $g: K^{\prime} \rightarrow K$ be the simplicial map obtained from the Hopf Approximation Theorem (cf. [1] p. 118). Since mesh $K^{\prime}<\varepsilon$, the map $g$ is expanding on those simplexes where it is nondegenerate. So $g$ is homotopic to $f$ and satisfies (i). It remains to show (ii).

Let $x \in|K|$ be a fixed point of $g^{n}$. Let $\sigma_{j}$ be the carrier of $g^{j}(x)$ in $K^{\prime}$, $j=0, \ldots, n$. Since $g\left(g^{j}(x)\right)=g^{j+1}(x)$ for $j<n$ and $g^{n}(x)=x$, we have $g\left(\sigma_{j}\right) \supset \sigma_{j+1}$ for $j<n$ and $\sigma_{n}=\sigma_{0}$. Hence each $g \mid \sigma_{j}$ does not decrease dimension (otherwise we can never get $\sigma_{n}=\sigma_{0}$ ), i.e. $g \mid \sigma_{j}$ is nondegenerate for $0 \leq j \leq n-1$. By the choice of $\varepsilon, g \mid \sigma_{j}$ is expanding for $0 \leq j \leq n-1$.

Let $\tau=\left(g \mid \sigma_{0}\right)^{-1}\left(g \mid \sigma_{1}\right)^{-1} \cdots\left(g \mid \sigma_{n-1}\right)^{-1} \sigma_{0}$, then $\tau$ is a simplex in $\sigma_{0}$ containing $x$. Since every $g \mid \sigma_{j}$ is expanding, $g^{n}: \tau \rightarrow \sigma_{0}$ is also expanding, hence $x$ is the only fixed point on $\tau$.

This analysis shows that no two fixed points of $g^{n}$ can share the same sequence $\sigma_{0}, \sigma_{1}, \ldots, \sigma_{n-1}$ of simplexes of $K^{\prime}$. (If they did, they would be in the same $\tau$, a contradiction.) But $K^{\prime}$ is a finite complex, there are only finitely many different sequences of simplexes of length $n$. Hence $g^{n}$ has only finitely many fixed points. 
3. Proof of the Main Theorem. (A) We will need the following result in [5], §3.2. See also [4].

Lemma. Let $f: X \rightarrow X$ be a map, $\tilde{f}: \tilde{X} \rightarrow \tilde{X}$ be a lifting of $f$. Suppose $f_{\pi}^{n} \pi_{1}(X)$ is abelian for some $n$. Then the composition

$$
\pi_{1}\left(X, x_{0}\right) \stackrel{\theta}{\rightarrow} H_{1}(X) \stackrel{\eta}{\rightarrow} \operatorname{Coker}\left(1-f_{*}: H_{1}(X) \rightarrow H_{1}(X)\right),
$$

where $\theta$ is the abelianization and $\eta$ is the natural projection, induces a bijection from the set of fixed point classes of $f$ onto Coker $\left(1-f_{*}\right)$, sending $p \operatorname{Fix}(\alpha \circ f)$ to $\eta \circ \theta(\alpha), \alpha \in \pi$.

In our Main Theorem, we assume $f_{\pi}^{n} \pi_{1}(X) \subset J\left(f^{n}\right)$ for some $n$, thus $f_{\pi}^{n} \pi_{1}(X)$ is abelian as mentioned in $\S 1$, and the Lemma applies. So the last sentence of the Main Theorem follows from the preceding one.

(B) The case $n=1$, namely $f_{\pi} \pi_{1}(X) \subset J(f)$.

As mentioned in $\S 1$, this is the same as $\tilde{f}_{\pi} \pi \subset J(\tilde{f})$. For any $\alpha \in \pi$, we always have $p \operatorname{Fix}(\alpha \circ \tilde{f})=p \operatorname{Fix}\left(\tilde{f}_{\pi}(\alpha) \circ \tilde{f}\right)$ since $\alpha$ is $\tilde{f}_{\pi}$-conjugate to $\tilde{f}_{\pi}(\alpha)$. Now $\tilde{f}_{\pi}(\alpha) \in \tilde{f}_{\pi} \pi \subset J(\tilde{f})$, so there is a homotopy $\left\{h_{t}\right\}: f \simeq f$ which lifts to $\tilde{f} \simeq \tilde{f}_{\pi}(\alpha) \circ \tilde{f}$. Hence by the homotopy invariance we have $\operatorname{index}(f, p \operatorname{Fix}(\tilde{f}))=\operatorname{index}(f, p \operatorname{Fix}(\alpha \circ \tilde{f}))$. This $\alpha$ is arbitrary, so that any two fixed point classes of $f$ have the same index.

(C) Pick a prime $q$ such that

(i) $f_{\pi}^{q} \pi_{1}(X) \subset J\left(f^{q}\right)$,

(ii) $q$ is coprime to the order of the torsion subgroup of Coker $\left(1-f_{*}\right)$, and

(iii) $q$ is larger than the absolute value of the difference of indices for any two fixed point classes of $f$.

Such a $q$ exists since if $n$ satisfies the condition in the hypothesis then so does any $n^{\prime}>n$.

Let $\mathbf{F}_{i}=p \operatorname{Fix}\left(\alpha_{i} \circ \tilde{f}\right), i=1,2$, be two fixed point classes of $f$. The respective fixed point classes of $f^{q}$ containing them are $\mathbf{F}_{i}^{(q)}=$ $p \operatorname{Fix}\left(\alpha_{i} \circ \tilde{f}\right)^{q}=p \operatorname{Fix}\left(\alpha_{i}^{(q)} \circ \tilde{f}^{q}\right)$, where

$$
\alpha_{i}^{(q)}=\alpha_{\imath} \tilde{f}_{\pi}\left(\alpha_{i}\right) \cdots \tilde{f}_{\pi}^{q-1}\left(\alpha_{i}\right), \quad i=1,2 .
$$

(D) Claim: Different fixed point classes of $f$ are contained in different fixed point classes of $f^{q}$.

Suppose $\mathbf{F}_{1}^{(\mathrm{q})}=\mathbf{F}_{2}^{(\mathrm{q})}$. We want to show $\mathbf{F}_{1}=\mathbf{F}_{2}$. In fact, we may assume $\alpha_{1}^{(q)}=\alpha_{2}^{(q)}$. Otherwise, since $\alpha_{1}^{(q)}=\alpha_{2}^{(q)}$ are $\tilde{f}_{\pi}^{q}$-conjugate there is a 
$\gamma$ such that $\alpha_{2}^{(q)}=\gamma \alpha_{1}^{(q)} \tilde{f}_{\pi}^{q}\left(\gamma^{-1}\right)$, and we may use $\alpha_{1}^{\prime}=\gamma \alpha_{1} \tilde{f}_{\pi}\left(\gamma^{-1}\right)$ in place of $\alpha_{1}$. Note that

$$
\begin{aligned}
\eta \circ \theta\left(\alpha_{i}^{(q)}\right) & =\eta \circ \theta\left(\alpha_{i} \tilde{f}_{\pi}\left(\alpha_{i}\right) \cdots \tilde{f}_{\pi}^{q-1}\left(\alpha_{i}\right)\right) \\
& =\eta\left(\theta\left(\alpha_{\imath}\right)+f_{*} \circ \theta\left(\alpha_{i}\right)+\cdots+f_{*}^{q-1} \circ \theta\left(\alpha_{i}\right)\right) \\
& =q \eta \circ \theta\left(\alpha_{i}\right) .
\end{aligned}
$$

So $q \eta \circ \theta\left(\alpha_{1}\right)=q \eta \circ \theta\left(\alpha_{2}\right)$. By the condition (ii) for $q$, we have $\eta \circ \theta\left(\alpha_{1}\right)$ $=\eta \circ \theta\left(\alpha_{2}\right)$. Now we see $\mathbf{F}_{1}=\mathbf{F}_{2}$ by $(\mathrm{A})$.

(E) Applying the result of (B) to the map $f^{q}: X \rightarrow X$, in view of the condition (i) for $q$, we get

$$
\operatorname{index}\left(f^{q}, \mathbf{F}_{1}^{(q)}\right)=\operatorname{index}\left(f^{q}, \mathbf{F}_{2}^{(q)}\right) .
$$

By (D) and the $\bmod p$ Index Theorem, $\left(f \mathbf{F}_{i}^{(q)}=\mathbf{F}_{i}^{(q)}\right.$ since $\left.\mathbf{F}_{l} \subset \mathbf{F}_{l}^{(q)}\right)$

$$
\operatorname{index}\left(f^{q}, \mathbf{F}_{i}^{(q)}\right) \equiv \operatorname{index}\left(f, \mathbf{F}_{l}\right) \quad \bmod q, \quad i=1,2 .
$$

Hence

$$
\operatorname{index}\left(f, \mathbf{F}_{1}\right) \equiv \operatorname{index}\left(f, \mathbf{F}_{2}\right) \quad \bmod q .
$$

But $q$ is large enough by condition (iii) so that we have

$$
\operatorname{index}\left(f, \mathbf{F}_{1}\right)=\operatorname{index}\left(f, \mathbf{F}_{2}\right) \text {. }
$$

\section{Corollaries.}

Corollary 1. Suppose $X$ is aspherical, $f: X \rightarrow X$ is such that $f_{\pi}^{n} \pi_{1}(X)$ is abelian for some $n$. Then $L(F)=0$ implies $N(f)=0$, while $L(f) \neq 0$ implies $N(f)=\# \operatorname{Coker}\left(1-f_{*}\right)$.

Proof. Since $X$ is aspherical, by Gottlieb's Theorem (cf. [1] p. 102),

$$
J\left(f^{n}\right)=Z\left(f_{\pi}^{n} \pi_{1}(X), \pi_{1}(X)\right) .
$$

But $f_{\pi}^{n} \pi_{1}(X)$ is abelian iff $f_{\pi}^{n} \pi_{1}(X) \subset Z\left(f_{\pi}^{n} \pi_{1}(X), \pi_{1}(X)\right)$. So we have $f_{\pi}^{n} \pi_{1}(X) \subset J\left(f^{n}\right)$ for some $n$. The conclusion now follows from the Main Theorem.

Corollary 2. Suppose $f: X \rightarrow X, \cap_{n=1}^{\infty} f^{n}(X)$ is contained in a subpolyhedron $X_{0} \subset X$ such that $J\left(X_{0}\right)=\pi_{1}\left(X_{0}\right)$. Then $L(f)=0$ implies $N(f)=0$, and $L(f) \neq 0$ implies $N(f)=\# \operatorname{Coker}\left(1-f_{*}\right)$. 
Proof. Replacing $X_{0}$ by a regular neighborhood if necessary, we may assume that there is an integer $n$ such that $f^{n} X \subset X_{0}$. Pick a fixed point $x_{0}$ of $f$ as base point. Write $\pi=\pi_{1}\left(X, x_{0}\right)$ and $\pi^{\prime}=\pi_{1}\left(X_{0}, x_{0}\right)$. Let $i_{\pi}$ : $\pi^{\prime} \rightarrow \pi$ be induced by the inclusion $i: X_{0} \rightarrow X$. Since $f^{n}$ can be factored through $i$, we have $f_{\pi}^{n} \pi \subset i_{\pi} \pi^{\prime}$ and $i_{\pi} J\left(X_{0}\right) \subset J\left(f^{n}\right)$. Thus

$$
f_{\pi}^{n} \pi \subset i_{\pi} \pi^{\prime}=i_{\pi} J\left(X_{0}\right) \subset J\left(f^{n}\right) .
$$

Now apply the Main Theorem.

5. Remarks. The following simple example shows that our Main Theorem is indeed better than the results in [3].

ExAmple. Let $X=\bigvee_{i=1}^{n} S_{i}$ be a bouquet of circles at $x_{0}$. Suppose a $\operatorname{map} f: X, x_{0} \rightarrow X, x_{0}$ satisfies

$$
\begin{array}{ll}
f\left(\alpha_{1}\right)=k \alpha_{1}, & (k \neq 0), \\
f\left(\alpha_{i}\right)=\alpha_{l-1} & \text { for } i>1,
\end{array}
$$

where $\alpha_{i}$ is a generator of $\pi_{1}\left(S_{i}, x_{0}\right) \subset \pi_{1}\left(X, x_{0}\right)$. Since $X$ is aspherical, by Gottlieb's Theorem we have

$$
J\left(f^{n}\right)=Z\left(f_{\pi}^{n}(\pi), \pi\right)= \begin{cases}0 & \text { if } n<m-1 \\ \pi_{1}\left(S_{i}, x_{0}\right) & \text { if } n \geq m-1\end{cases}
$$

So that $f_{\pi}^{n}(\pi) \not \subset J\left(f^{n}\right)$ for $n<m-1$, but $f_{\pi}^{n}(\pi) \subset J\left(f^{n}\right)$ for $n \geq$ $m-1$.

Actually all the computations in [1], VII.C are easy consequences of the Main Theorem.

As mentioned in the introduction, a nice feature of our Main Theorem is that the hypothesis is symmetric. More precisely,

Proposition. Let $\varphi: X \rightarrow Y, \psi: Y \rightarrow X, f=\psi \circ \varphi$ and $g=\varphi \circ \psi$. Then $f_{\pi}^{n} \pi_{1}(X) \subset J\left(f^{n}\right)$ for some $n$ iff $g_{\pi}^{n} \pi_{1}(Y) \subset J\left(g^{n}\right)$ for some $n$.

Proof. Let $x_{0}$ be a fixed point of $f$ and $y_{0}=\varphi\left(x_{0}\right)$. Then

$$
g_{\pi}^{n+1} \pi_{1}\left(Y, y_{0}\right)=\varphi_{\pi} \circ f_{\pi}^{n} \circ \psi_{\pi} \pi_{1}\left(Y, y_{0}\right) \subset \varphi_{\pi} \circ f_{\pi}^{n} \pi_{1}\left(X, x_{0}\right) .
$$

On the other hand, we have $\varphi_{\pi} J\left(f^{n}, x_{0}\right) \subset J\left(g^{n+1}, y_{0}\right)$ as mentioned in $\S 1$. Hence $f_{\pi}^{n} \pi_{1}(X) \subset J\left(f^{n}\right)$ implies $g_{\pi}^{n+1} \pi_{1}(Y) \subset J\left(g^{n+1}\right)$. 


\section{REFERENCES}

1. R. F. Brown, The Lefschetz Fixed Point Theorem, Scott, Foresman \& Company, Glenview, Illinois, 1971.

2. R. Geoghegan, The homomorphism on fundamental group induced by a homotopy idempotent having essential fixed points, preprint, 1979.

3. B. Jiang, Estimation of the Nielsen numbers, Chinese Math-Acta, 5 (1964), 330-339.

4. L_Lectures on Nielsen Fixed Point Theory, in preparation.

5. T. H. Kiang, Theory of Fixed Point Classes, Scientific Press, Peking, 1979.

Received September 29, 1981.

UNIVERSITY OF CALIFORNIA

Los ANGeles, CA 90024

Current address: Peking University

Peking, China 



\section{PACIFIC JOURNAL OF MATHEMATICS EDITORS}

DONALD BABBITT (Managing Editor)

University of California

Los Angeles, CA 90024

Hugo Rossi

University of Utah

Salt Lake City, UT 84112

C. C. Moore and Arthur Ogus

University of California

Berkeley, CA 94720
J. DugunduI

Department of Mathematics

University of Southern California

Los Angeles, CA 90089-1113

R. FinN and H. SAmelson

Stanford University

Stanford, CA 94305

\section{ASSOCIATE EDITORS}
R. ARENS
E. F. BECKENBACH
B. H. NeUmaNN
F. WolF
K. YoshidA (1906-1982)

\section{SUPPORTING INSTITUTIONS}

UNIVERSITY OF ARIZONA

UNIVERSITY OF BRITISH COLUMBIA

CALIFORNIA INSTITUTE OF TECHNOLOGY

UNIVERSITY OF CALIFORNIA

MONTANA STATE UNIVERSITY

UNIVERSITY OF NEVADA, RENO

NEW MEXICO STATE UNIVERSITY

OREGON STATE UNIVERSITY
UNIVERSITY OF OREGON

UNIVERSITY OF SOUTHERN CALIFORNIA

STANFORD UNIVERSITY

UNIVERSITY OF HAWAII

UNIVERSITY OF TOKYO

UNIVERSITY OF UTAH

WASHINGTON STATE UNIVERSITY

UNIVERSITY OF WASHINGTON 


\section{Pacific Journal of Mathematics}

\section{Vol. 106, No. $1 \quad$ November, 1983}

John Ballard, Clifford's theorem for algebraic groups and Lie algebras . . . . . 1

Philip Richard Bartick, II and Edwin Duda, Finite-to-one open mappings

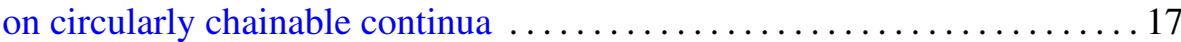

Frank Hayne Beatrous, Jr., $H^{\infty}$-interpolation from a subset of the

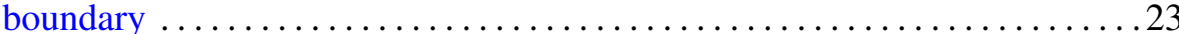

Sterling K. Berberian, Expectations in semifinite algebras ............ 33

Heron S. Collins and Wolfgang Ruess, Weak compactness in spaces of compact operators and of vector-valued functions $\ldots \ldots \ldots \ldots \ldots \ldots . \ldots 45$

David Downing and William O. Ray, Renorming and the theory of

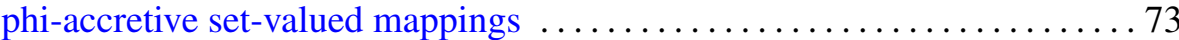

Harvey Charles Greenwald, On the theory of homogeneous Lipschitz

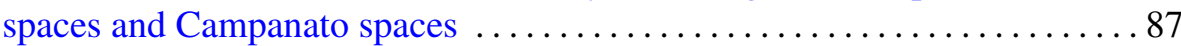

Irwen Valle Guadalupe and Lucio Ladislao Rodriguez, Normal curvature

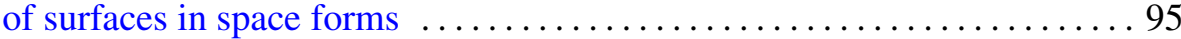

Boju Jiang, On the computations of the Nielsen number ............... 105

William H. Julian, Ray Mines, III and Fred Richman, Alexander

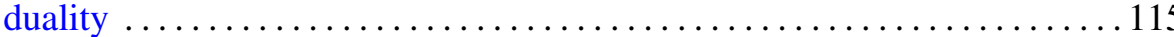

Midori Kobayashi, The connected component of the idèle class group of an

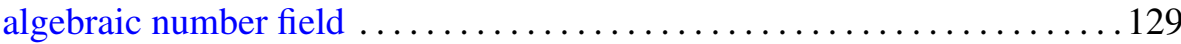

Ib Henning Madsen, Charles B. Thomas and C. Terence C. (Charles)

(Clegg) Wall, Topological spherical space form problem. III.

Dimensional bounds and smoothing

A. Sapounakis, The existence of strong liftings for totally ordered measure

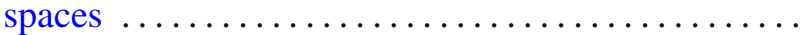

Gary Seitz, The root subgroups for maximal tori in finite groups of Lie type

Laurent Siebenmann and James M. Van Buskirk, Construction of irreducible homology 3-spheres with orientation reversing involution 